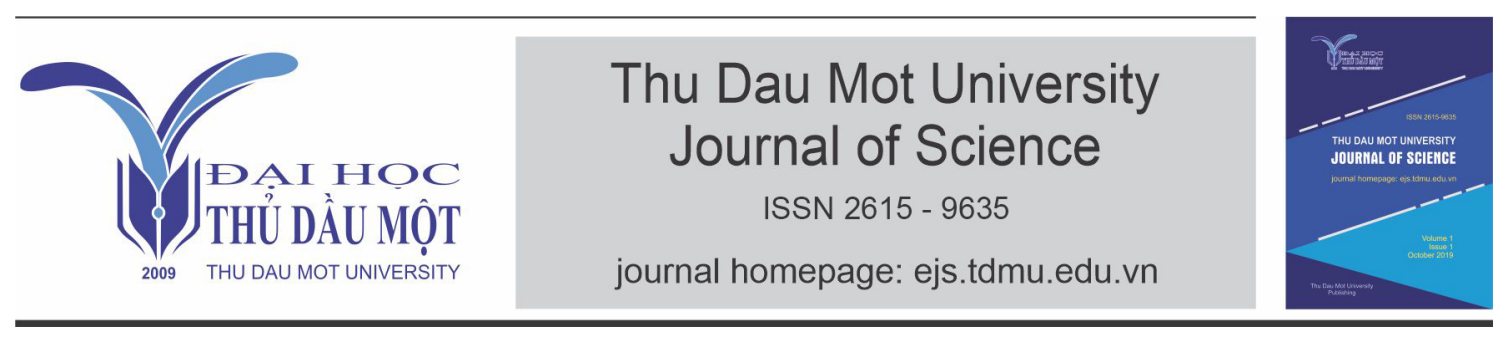

\title{
A new class of bilevel weak vector variational in- equality problems
}

by Nguyen Van Hung (Posts and Telecommunications Institute of Technology Ho Chi Minh City), Vo Viet Tri (Thu Dau Mot university)

Article Info: Received Sep. 15 $5^{\text {th }}$, 2020, Accepted Nov. 4 ${ }^{\text {th }}$, 2020, Available online Dec. $15^{\text {th }}, 2020$

Corresponding author: trivv@tdmu.edu.vn (Vo Viet Tri)

https://doi.org/10.37550/tdmu.EJS/2020.04.078

\begin{abstract}
In this paper, we first introduce a new class of bilevel weak vector variational inequality problems in locally convex Hausdorff topological vector spaces. Then, using the Kakutani-Fan-Glicksberg fixed-point theorem, we establish some existence conditions of the solution for this problem.
\end{abstract}

Keywords: Bilevel weak vector variational inequality problems; KakutaniFan-Glicksberg fixed-point theorem; existence conditions

\section{Introduction and Preliminaries}

It is known that, the existence conditions of solutions of optimization-related problems is one of the important topics in optimization theory and so many authors have tried to find several good conditions of the existence of solution sets of various problems as optimization problems, complementarity problems traffic network problems, equilibrium problems $[5,8,9]$ and the references therein.

On the other hand, Mordukhovich [12] introduced equilibrium problems with equilibrium constraints and studied optimal conditions to this problem in 2004. In 
recent years, equilibrium problems with equilibrium constraints have been attracted by many authors in different directions, for example, the existence conditions of solutions $[3,6,10]$, the stability properties of solutions $[6,7,2]$. However, to the best of our knowledge, up to now, there have not been any works on the existence conditions of solutions of bilevel weak vector variational inequality problems.

Motivated and inspired by the above, in this paper, we investigate the existence conditions of solutions for bilevel weak vector variational inequality problems in locally convex Hausdorff topological vector spaces. Let $X, Z$ be real locally convex Hausdorff topological vector spaces, $L(X, Z)$ be the space of all linear continuous operators from $X$ into $Z, A$ be a nonempty compact subset of $X$ and $C_{1} \subset Z$ be a closed convex and pointed cone with $\operatorname{int} C_{1} \neq \emptyset$, where $\operatorname{int} C_{1}$ is the interior of $C_{1}$. Let $K: A \rightrightarrows A$ and $T: A \rightrightarrows L(X, Z)$ be multifunctions, $\eta: A \times A \rightarrow A$ be a continuous single-valued mapping. Denoted $\langle z, x\rangle$ by the value of a linear operator $z \in L(X ; Y)$ at $x \in A$, we always assume that $\langle.,\rangle:. L(X ; Z) \times A \rightarrow Z$ is continuous. We consider the following weak vector quasi-variational inequality problems:

(WQVIP) Find $x \in A$ such that, there exists $z \in T(x)$ satisfying

$$
\left\{\begin{array}{l}
x \in K(x) \\
\langle z, \eta(y, x)\rangle \in Z \backslash-\operatorname{int} C_{1} \text { for all } y \in K(x) .
\end{array}\right.
$$

We denote the solution set of the problem (WQVIP) by $\mathbb{Q}(K, T)$.

Let $P$ be a real locally convex Hausdorff topological vector space, $L(X, P)$ be the space of all linear continuous operators from $X$ into $P, C_{2} \subset P$ be a closed convex and pointed cone with int $C_{2} \neq \emptyset$ and $H: A \rightarrow L(X, P)$ be a single-valued mapping.

Also, we consider the following weak bilevel vector variational inequality problems: (WBVIP) Find a point $x \in \mathbb{Q}(K, T)$ such that

$$
\langle H(x), y-x\rangle \in P \backslash-\operatorname{int} C_{2}, \quad \forall y \in \mathbb{Q}(K, T) ;
$$

where $\mathbb{Q}(K, T)$ be the solution set of the weak vector quasi-variational inequality problems. We denote the solution set of the problem (WBVIP) by $\mathbb{O}(H)$.

Now, we recall the following well-known definitions and some results for the main results:

Definition 1.1 (see [1]) Let $X, Y$ be two topological vector spaces, $F: X \rightrightarrows Y$ be a multifunction and let $x_{0} \in X$ be a given point. 
(1) $F$ is said to be lower semi-continuous (lsc) at $x_{0} \in X$ if $F\left(x_{0}\right) \cap U \neq \emptyset$ for some open set $U \subseteq Y$ implies the existence of a neighborhood $N$ of $x_{0}$ such that $F(x) \cap U \neq \emptyset$ for all $x \in N$.

(2) $F$ is said to be upper semi-continuous (usc) at $x_{0} \in X$ if, for each open set $U \supseteq G\left(x_{0}\right)$, there is a neighborhood $N$ of $x_{0}$ such that $U \supseteq F(x)$ for all $x \in N$.

(3) $F$ is said to be continuous at $x_{0} \in X$ if it is both lsc and usc at $x_{0} \in X$

(4) $F$ is said to be closed at $x_{0}$ if, for each of the nets $\left\{x_{\alpha}\right\}$ in $X$ converging to $x_{0}$ and $\left\{y_{\alpha}\right\}$ in $Y$ converging to $y_{0}$ such that $y_{\alpha} \in F\left(x_{\alpha}\right)$, we have $y_{0} \in F\left(x_{0}\right)$.

If $A \subset X$, then $F$ is said to be usc (lsc, continuous, closed, respectively) on the set $A$ if $F$ is usc (lsc, continuous, closed, respectively) at all $x \in \operatorname{dom} F \cap A$. If $A \equiv X$, then we omit "on $X$ " in the statement.

Lemma 1.1 (see [1]) Let $X, Y$ be two topological vector spaces and $F: X \rightrightarrows Y$ be a multifunction. Then we have the following:

(1) If $F$ is upper semi-continuous with closed values, then $F$ is closed.

(2) If $F$ is closed and $F(X)$ is compact, then $F$ is upper semi-continuous.

Lemma 1.2 (see [1]) Let $X, Y$ be two topological vector spaces and $F: X \rightrightarrows Y$ be a multifunction. Then we have the following:

(1) $F$ is lower semi-continuous $x_{0} \in X$ if and only if, for each net $\left\{x_{\alpha}\right\} \subseteq X$ which converges to $x_{0} \in X$ and for each $y_{0} \in F\left(x_{0}\right)$, there exists $\left\{y_{\alpha}\right\}$ in $Y$ such that $y_{\alpha} \in F\left(x_{\alpha}\right), y_{\alpha} \rightarrow y_{0}$.

(2) If $F$ has compact values, then $F$ is upper semi-continuous $x_{0} \in X$ if and only if, for each net $\left\{x_{\alpha}\right\} \subseteq X$ which converges to $x_{0} \in X$ and for each net $\left\{y_{\alpha}\right\}$ in $Y$ such that $y_{\alpha} \in F\left(x_{\alpha}\right)$, there exist $y_{0} \in F\left(x_{0}\right)$ and a subnet $\left\{y_{\beta}\right\}$ of $\left\{y_{\alpha}\right\}$ such that $y_{\beta} \rightarrow y_{0}$.

Lemma 1.3 (see [4]) Let $A$ be a nonempty convex compact subset of Hausdorff topological vector space $X$ and $N$ be a subset of $A \times A$ such that 
(i) for each at $x \in A,(x, x) \notin N$;

(ii) for each at $y \in A$, the set $\{x \in A:(x, y) \in N\}$ is open on $A$;

(iii) for each at $x \in A$, the set $\{y \in A:(x, y) \in N\}$ is convex or empty.

Then there exists $x_{0} \in A$ such that $\left(x_{0}, y\right) \notin N$ for all $y \in A$.

Lemma 1.4 (see [11]) Let $A$ be a nonempty compact convex subset of a locally convex Hausdorff vector topological space $X$. If $F: A \rightrightarrows A$ is upper semi-continuous and, for any $x \in A, F(x)$ is nonempty convex closed, then there exists $x^{*} \in A$ such that $x^{*} \in F\left(x^{*}\right)$.

\section{$2 \quad$ Main Results}

In this section, we establish some existence results for weak bilevel vector quasivariational inequality problems.

We first introduce the concept of weakly $C$-quasiconvexity.

Definition 2.1 Let $X, Z$ be two topological vector spaces, $A$ be a nonempty closed subset of $X$, and $C \subset Z$ is a solid pointed closed convex cone and $f: A \rightarrow Z$ be a function.The mapping $f$ is said to be weakly $C$-quasiconvex on $A \subset X$ if, for each $x_{1}, x_{2} \in A, \lambda \in[0,1]$ with $f\left(x_{1}\right) \in Z \backslash-\operatorname{int} C$ and $f\left(x_{2}\right) \in Z \backslash-\operatorname{int} C$, we have

$$
f\left((1-\lambda) x_{1}+\lambda x_{2}\right) \in Z \backslash-\operatorname{int} C,
$$

We now establish some existence conditions of solution sets of the weak vector quasi-variational inequality problems.

Lemma 2.1 Let $X, Z$ be real locally convex Hausdorff topological vector spaces, $L(X, Z)$ be the space of all linear continuous operators from $X$ into $Z, A$ be a nonempty compact subset of $X$ and $C_{1} \subset Z$ be a closed convex and pointed cone with $\operatorname{int} C_{1} \neq \emptyset$, where int $C_{1}$ is the interior of $C_{1}$. Let $K: A \rightrightarrows A$ and $T: A \rightrightarrows L(X, Z)$ be multifunctions, $\eta: A \times A \rightarrow A$ be a continuous single-valued mapping. Denoted $\langle z, x\rangle$ by the value of a linear operator $z \in L(X ; Z)$ at $x \in A$, we always assume that $\langle.,\rangle:. L(X ; Z) \times A \rightarrow Z$ is continuous. Suppose the following conditions:

(i) $K$ is continuous on $A$ with nonempty compact convex values; 
(ii) $T$ is upper semicontinuous on $A$ with nonempty compact values;

(iii) for all $x \in A, z \in L(X ; Z),\langle z, \eta(x, x)\rangle \in Z \backslash-\operatorname{int} C_{1}$;

(iv) for all $x \in A, z \in L(X ; Z)$, the set $\left\{y \in A:\langle z, \eta(y, x)\rangle \notin Z \backslash-\operatorname{int} C_{1}\right\}$ is convex;

(v) for all $y \in A, z \in L(X ; Z)$, the map $x \mapsto\langle z, \eta(y, x)\rangle$ is weakly $C_{1}$-quasiconvex, i.e., for all $x_{1}, x_{2} \in A$ and all $\lambda \in[0,1], y \in A, z \in L(X ; Z)$, we have

$$
\begin{aligned}
& \left\langle z, \eta\left(y, x_{1}\right)\right\rangle \in Z \backslash-\operatorname{int} C_{1} \text { and }\left\langle z, \eta\left(y, x_{2}\right)\right\rangle \in Z \backslash-\operatorname{int} C_{1} \\
& \Longrightarrow\left\langle z, \eta\left(y, \lambda x_{1}+(1-\lambda) x_{2}\right)\right\rangle \in Z \backslash-\operatorname{int} C_{1}
\end{aligned}
$$

(vi) the set $\left\{(x, y, z) \in A \times A \times L(X, Z):\langle z, \eta(y, x)\rangle \in Z \backslash-\operatorname{int} C_{1}\right\}$ is closed.

Then the weak vector quasi-variational inequality problem has a solution, i.e., there exist $\bar{x} \in A$ and $\bar{z} \in T(\bar{x})$ such that $\bar{x} \in K(\bar{x})$ satisfying

$$
\langle\bar{z}, \eta(y, \bar{x})\rangle \in Z \backslash-\operatorname{int} C_{1}, \forall y \in K(\bar{x}) .
$$

Moreover, the solution set of the weak vector quasi-variational inequality problem is compact.

Proof. For all $x \in A, z \in L(X, Z)$, we define a multifunction $\mathbb{M}: A \times L(X, Z) \rightrightarrows$ $A$ by

$$
\mathbb{M}(x, z)=\left\{a \in K(x):\langle z, \eta(y, a)\rangle \in Z \backslash-\operatorname{int} C_{1}, \forall y \in K(x)\right\}
$$

First, we show that $\mathbb{M}(x, z)$ is nonempty. Indeed, for every $x \in A, K(x)$ is nonempty compact convex set. Set

$$
\mathbb{N}=\left\{(a, y) \in K(x) \times K(x):\langle z, \eta(y, a)\rangle \notin Z \backslash-\operatorname{int} C_{1}\right\}
$$

By the condition (iii), we have for any $a \in K(x),(a, a) \in \mathbb{N}$. It follows from the condition (iv) that the set $\{y \in K(x):(a, y) \notin \mathbb{N}\}$ is convex. Moreover, by the condition (iv), we have for any $a \in K(x)$, the set $\{y \in K(x):(a, y) \in \mathbb{N}\}$ is open. So, by Lemma 1.3 there exists $a^{*} \in K(x)$ such that $\left(a^{*}, y\right) \notin \mathbb{N}$, for all $y \in K(x)$, i.e.,

$$
\left\langle z, \eta\left(y, a^{*}\right)\right\rangle \in Z \backslash-\operatorname{int} C_{1}, \forall y \in K(x) .
$$

Hence, $\mathbb{M}(x, z)$ is nonempty. 
Second, we verify that $\mathbb{M}(x, z)$ is a convex set. In fact, let $a_{1}, a_{2} \in \mathbb{M}(x, z)$, $\lambda \in[0,1]$ and put $a=\lambda a_{1}+(1-\lambda) a_{2}$. Since $a_{1}, a_{2} \in K(x)$ and $K(x)$ is a convex set, we have $a \in K(x)$. From $a_{1}, a_{2} \in \mathbb{M}(x, z)$, it follows that, for any $y \in K(x)$, we have

$$
\left\langle z, \eta\left(y, a_{1}\right)\right\rangle \in Z \backslash-\operatorname{int} C_{1} \text { and }\left\langle z, \eta\left(y, a_{2}\right)\right\rangle \in Z \backslash-\operatorname{int} C_{1} .
$$

By the condition $(\mathrm{v})$, since the map $x \mapsto\langle z, \eta(y, x)\rangle$ is weakly $C_{1}$-quasiconvex, we have

$$
\left\langle z, \eta\left(y, \lambda x_{1}+(1-\lambda) x_{2}\right)\right\rangle \in Z \backslash-\operatorname{int} C_{1}, \quad \forall \lambda \in[0,1],
$$

i.e., $a \in \mathbb{M}(x, z)$. Therefore, $\mathbb{M}(x, z)$ is convex.

Third, we prove that $\mathbb{M}$ is upper semi-continuous with compact values. Indeed, since $A$ is a compact set, by Lemma 1.1(ii), we need only to show that $\mathbb{M}$ is a closed mapping. In fact, assume that a net $\left\{\left(x_{\alpha}, z_{\alpha}, a_{\alpha}\right)\right\} \subset A \times L(X, Z) \times K(x)$ with $a_{\alpha} \in \mathbb{M}\left(x_{\alpha}, z_{\alpha}\right)$ such that $x_{\alpha} \rightarrow x \in A, z_{\alpha} \rightarrow z \in L(X, Z)$ and $a_{\alpha} \rightarrow a_{0}$.

Now, we need to verify that $a_{0} \in \mathbb{M}(x, z)$. Since $a_{\alpha} \in K\left(x_{\alpha}\right)$ and $K$ is upper semi-continuous on $A$ with nonempty compact values, it follows that $K$ is closed and so we have $a_{0} \in K(x)$. Suppose that $a_{0} \notin \mathbb{M}(x, z)$. There exists $y_{0} \in K(x)$ such that

$$
\left\langle z_{0}, \eta\left(y_{0}, a_{0}\right)\right\rangle \notin-\operatorname{int} C_{1} .
$$

It follows from the lower semi-continuity of $K$ that there is a net $\left\{y_{\alpha}\right\}$ such that $y_{\alpha} \in K\left(x_{\alpha}\right)$ and $y_{\alpha} \rightarrow y_{0}$ (taking a subnet if necessary). Since $a_{\alpha} \in \mathbb{M}\left(x_{\alpha}, z_{\alpha}\right)$, we have

$$
\left\langle z_{\alpha}, \eta\left(y_{\alpha}, a_{\alpha}\right)\right\rangle \in Z \backslash-\operatorname{int} C_{1} \text { for all } \alpha
$$

By the condition (vi) together with (2.2), it follows that

$$
\left\langle z, \eta\left(y_{0}, a_{0}\right)\right\rangle \in Z \backslash-\operatorname{int} C_{1}
$$

This is the contradiction from (2.1) and (2.3). Therefore, we conclude that $a_{0} \in$ $\mathbb{M}(x, z)$. Hence $\mathbb{M}$ is upper semi-continuous with nonempty compact values.

Fourth, we need to prove the solution set $\mathbb{Q}(K, T) \neq \emptyset$.

Define the set-valued mapping $\Psi: A \times L(X, Z) \rightrightarrows A \times L(X, Z)$ by

$$
\Psi(x, z)=(\mathbb{M}(x, z), T(x)), \forall(x, z) \in A \times L(X, Z) .
$$

Then, $\Psi$ is upper semicontinuous on $A \times L(X, Z), \Psi(x, z)$ is nonempty closed convex subset of $A \times L(X, Z)$. By Lemma 1.4, there exists a point $(x, z) \in A \times L(X, Z)$ such 
that $(x, z) \in \Psi(x, z)$, i.e., $x \in \mathbb{M}(x, z), z \in T\left(x^{*}\right)$. This implies that $(x, z) \in A \times T(x)$ satisfy $x \in K(x)$ and

$$
\langle z, \eta(y, x)\rangle \in Z \backslash-\operatorname{int} C_{1}, \forall y \in K(x)
$$

i.e., the weak vector quasi-variational inequality problem has a solution.

Finally, we prove that $\mathbb{Q}(K, T)$ is compact. In fact, since $A$ is compact and $\mathbb{Q}(K, T) \subset A$, we need only prove that $\mathbb{Q}(K, T)$ is closed. Indeed, let a net $\left\{x_{\alpha}\right\} \subset$ $\mathbb{Q}(K, T)$ be such that $x_{\alpha} \rightarrow x_{0}$. Now, we prove that $x_{0} \in \mathbb{Q}(K, T)$.

For any $y_{0} \in K\left(x_{0}\right)$, it follows from the lower semi-continuity of $K$, there is a net $\left\{y_{\alpha}\right\} \subset A$ with $y_{\alpha} \in K\left(x_{\alpha}\right)$ and $y_{\alpha} \rightarrow y_{0}$. Since $x_{\alpha} \in \mathbb{Q}(K, T)$, there exists $z_{\alpha} \in T\left(x_{\alpha}\right)$ such that

$$
\left\langle z_{\alpha}, \eta\left(y_{\alpha}, x_{\alpha}\right)\right\rangle \in Z \backslash-\operatorname{int} C_{1} \text { for all } \alpha \text {. }
$$

It follows from the upper semi-continuity and compactness $T$ that $z_{0} \in T\left(x_{0}\right)$ such that $z_{\alpha} \rightarrow z_{0}$ (taking subnets if necessary). By the condition (v) together with $\left(x_{\alpha}, y_{\alpha}, z_{\alpha}\right) \rightarrow\left(x_{0}, y_{0}, z_{0}\right)$, we have

$$
\left\langle z_{0}, \eta\left(y_{0}, x_{0}\right)\right\rangle \in Z \backslash-\operatorname{int} C_{1}
$$

this means that $x_{0} \in \mathbb{Q}(K, T)$. Thus $\mathbb{Q}(K, T)$ is a closed set. Therefore, $\mathbb{Q}(K, T)$ is compact. This completes the proof.

We now investigate the existence conditions for the weak bilevel vector variational inequality problems.

Theorem 2.1 Suppose that all the conditions in Lemma 2.1 are satisfied, $\mathbb{Q}(K, T)$ ) is convex. Let $P$ be a real locally convex Hausdorff topological vector space, $L(X, P)$ be the space of all linear continuous operators from $X$ into $P, C_{2} \subset P$ be a closed convex and pointed cone with $\operatorname{int} C_{2} \neq \emptyset$ and $H: A \rightarrow L(X, P)$ be a single-valued convex mapping. Denoted $\langle z, x\rangle$ by the value of a linear operator $z \in L(X ; P)$ at $x \in A$, we always assume that $\langle.,\rangle:. L(X ; P) \times A \rightarrow P$ is continuous and the following additional conditions:

(i') for all $x \in \mathbb{Q}(K, T),\langle H(x), x-x\rangle \in P \backslash-\operatorname{int} C_{2}$;

(ii') the set $\left\{y \in \mathbb{Q}(K, T):\left\langle H(x), y^{*}-x\right\rangle \in-\operatorname{int} C_{2}\right\}$ is convex; 
(iii') for all $y \in \mathbb{Q}(K, T)$, the map $x \mapsto\langle H(x), y-x\rangle$ is weakly $C_{2}$-quasiconvex, i.e., for all $x_{1}, x_{2} \in \mathbb{Q}(K, T)$ and all $\lambda \in[0,1], y \in \mathbb{Q}(K, T)$, we have

$$
\begin{aligned}
& \left\langle H\left(x_{1}\right), y-x_{1}\right\rangle \in P \backslash-\operatorname{int} C_{2} \text { and }\left\langle H\left(x_{1}\right), y-x_{1}\right\rangle \in P \backslash-\operatorname{int} C_{2} \\
& \Longrightarrow\left\langle H\left(\lambda x_{1}+(1-\lambda) x_{2}\right), y-\left(\lambda x_{1}+(1-\lambda) x_{2}\right)\right\rangle \in P \backslash-\operatorname{int} C_{2} ;
\end{aligned}
$$

(iv') the set $\left\{(x, y) \in \mathbb{Q}(K, T) \times \mathbb{Q}(K, T):\langle H(x), y-x\rangle \in P \backslash-\operatorname{int} C_{2}\right\}$ is closed.

Then the weak bilevel vector variational inequality problem has a solution, i.e., there exists $\bar{x} \in A$ such that $\bar{x} \in \mathbb{Q}(K, T)$ and

$$
\langle H(x), y-x\rangle \in P \backslash-\operatorname{int} C_{2}, \quad \forall y \in \mathbb{Q}(K, T) .
$$

Moreover, the solution set of the weak bilevel vector variational inequality problem is compact.

Proof. We define a multifunction $\mathbb{B}: A \rightrightarrows A$ by

$$
\mathbb{B}(x)=\left\{b \in \mathbb{Q}(K, T) \mid\langle H(b), y-b\rangle \in P \backslash-\operatorname{int} C_{2}, \quad \forall y \in \mathbb{Q}(K, T)\right\}, \quad x \in A
$$

First, we prove that $\mathbb{B}(x)$ is nonempty. Indeed, for all $y \in A, \mathbb{Q}(K, T)$ is a nonempty compact convex set. Set

$$
\mathbb{P}=\left\{(b, y) \in \mathbb{Q}(K, T) \times \mathbb{Q}(K, T):\langle H(b), y-b\rangle \in-\operatorname{int} C_{2}\right\} .
$$

Then we have the following: (a) The condition (i') implies that, for any $b \in \mathbb{Q}(K, T)$, $(b, b) \notin \mathbb{P}$. (b) The condition (ii') implies that, for any $b \in \mathbb{Q}(K, T),\{y \in A:(b, y) \in$ $\mathbb{P}\}$ is convex on $\mathbb{Q}(K, T)$. (c) The condition (iv') implies that, for any $b \in \mathbb{Q}(K, T)$, $\{y \in \mathbb{Q}(K, T):(b, y) \in \mathbb{P}\}$ is open on $\mathbb{Q}(K, T)$. By Lemma 1.3, there exists $b \in \mathbb{Q}(K, T)$ such that $(b, y) \notin \mathbb{P}$ for all $y \in \mathbb{Q}(K, T)$, i.e., $\langle H(b), y-b\rangle \in P \backslash-\operatorname{int} C_{2}$ for all $y \in \mathbb{Q}(K, T)\}$. Thus it follows that $\mathbb{B}(x)$ is nonempty.

Second, we show that $\mathbb{B}(x)$ is a convex set. In fact, let $b_{1}, b_{2} \in \mathbb{B}(x)$ and $\lambda \in[0,1]$ and put $b=\lambda b_{1}+(1-\lambda) b_{2}$. Since $b_{1}, b_{2} \in \mathbb{Q}(K, T)$ and $\mathbb{Q}(K, T)$ is a convex set, we have $b \in \mathbb{Q}(K, T)$. Thus it follows that, for all $b_{1}, b_{2} \in \mathbb{B}(x)$,

$$
\left\langle H\left(b_{1}\right), y-b_{1}\right\rangle \in P \backslash-\operatorname{int} C_{2} ; \text { and }\left\langle H\left(b_{2}\right), y-b_{2}\right\rangle \in P \backslash-\operatorname{int} C_{2}, \quad \forall y \in \mathbb{B}(x) .
$$

By the condition (iii'), since $x \mapsto\langle H(x), y-x\rangle$ is weakly $C_{2}$-quasiconvex, we have

$$
\left\langle H\left(\lambda b_{1}+(1-\lambda) b_{2}\right), y-\lambda b_{1}+(1-\lambda) b_{2}\right\rangle \in P \backslash-\operatorname{int} C_{2}, \quad \forall \lambda \in[0,1],
$$


i.e., $b \in \mathbb{B}(x)$. Thus, $\mathbb{B}(x)$ is convex.

Third, we prove that $\mathbb{B}$ is upper semi-continuous on $A$ with compact values. Indeed, since $A$ is a compact set, by Lemma 1.1 (ii), we need only to show that $\mathbb{B}$ is a closed mapping. Let a net $\left\{x_{\alpha}\right\} \subset A$ be such that $x_{\alpha} \rightarrow x \in A$ and let $b_{\alpha} \in \mathbb{B}\left(x_{\alpha}\right)$ be such that $b_{\alpha} \rightarrow b_{0}$.

Now, we need to show that $b_{0} \in \mathbb{B}(x)$. Since $b_{\alpha} \in \mathbb{Q}(K, T)$ and $\mathbb{Q}(K, T)$ is compact, we have $b_{0} \in \mathbb{Q}(K, T)$. Suppose that $b_{0} \notin \mathbb{B}(x)$. Then there exists $y \in \mathbb{Q}(K, T)$ such that

$$
\left\langle H\left(b_{0}\right), y-b_{0}\right\rangle \in-\operatorname{int} C_{2} .
$$

On the other hand, since $b_{\alpha} \in \mathbb{B}\left(x_{\alpha}\right)$, we have

$$
\left\langle H\left(b_{\alpha}\right), y-b_{\alpha}\right\rangle \in P \backslash-\operatorname{int} C_{2} \text { for all } \alpha .
$$

By the condition (iv') together with (2.5), it follows that

$$
\left\langle H\left(b_{0}\right), y-b_{0}\right\rangle \in P \backslash-\operatorname{int} C_{2},
$$

which is a contradiction from (2.4) and (2.6). Thus $b_{0} \in \mathbb{B}(x)$. Hence $\mathbb{B}$ is upper semi-continuous on $A$ with nonempty compact values.

Fourth, we prove that the solution set $\mathbb{O}(H)$ is nonempty. In fact, since $\mathbb{B}$ is upper semi-continuous on $A$ with nonempty compact values, by Lemma 1.4 , there exists a point $\hat{x} \in A$ such that $\hat{x} \in \mathbb{B}(\hat{x})$. Hence there exists $\hat{x} \in \mathbb{Q}(K, T)$ such that

$$
\langle H(\hat{x}), y-\hat{x}\rangle \in P \backslash-\operatorname{int} C_{2}, \quad \forall y \in \mathbb{Q}(K, T),
$$

i.e., the problem (WBVIP) has a solution.

Finally, we prove that $\mathbb{O}(H)$ is compact. Indeed, let a net $\left\{x_{\alpha}\right\} \subset \mathbb{O}(H)$ be such that $x_{\alpha} \rightarrow x_{0}$. Now, we prove that $x_{0} \in \mathbb{O}(H)$. By the closedness of $\mathbb{Q}(K, T)$, we have $x_{0} \in \mathbb{Q}(K, T)$. Since $x_{\alpha} \in \mathbb{O}(H)$, we obtain $x_{\alpha} \in \mathbb{Q}(K, T)$ and

$$
\left\langle H\left(x_{\alpha}\right), y-x_{\alpha}\right\rangle \in P \backslash-\operatorname{int} C_{2}, \quad \forall y \in \mathbb{Q}(K, T) .
$$

By the condition (iv') together with $x_{\alpha} \rightarrow x_{0}$, it follows that

$$
\left\langle H\left(x_{0}\right), y-x_{0}\right\rangle \in P \backslash-\operatorname{int} C_{2}, \forall y \in \mathbb{Q}(K, T),
$$

which means that $x_{0} \in \mathbb{O}(H)$. Thus $\mathbb{O}(H)$ is a closed set. Since $\mathbb{O}(H) \subset \mathbb{Q}(K, T)$ and $\mathbb{Q}(K, T)$ is compact. It follows that $\mathbb{O}(H)$ is compact subset of $A$. This completes the proof. 


\section{Conclusions}

In this work, we have established existence conditions to a new class of bilevel weak vector variational inequality problems. To the best of our knowledge, up to now, there have not been any works on the existence conditions of solutions for bilevel weak vector variational inequality problems by using the Kakutani-Fan-Glicksberg fixed-point theorem. Thus our results, Theorem 2.1 is new.

\section{Acknowledgements}

The authors wish to thank the anonymous referees for their valuable comments. This research is funded by Thu Dau Mot University, Binh Duong province, Viet Nam.

\section{References}

[1] Aubin, J.P,, Ekeland, I., Applied Nonlinear Analysis. John Wiley and Sons, New York, 1984

[2] Anh, L.Q., Hung, N.V.: Stability of solution mappings for parametric bilevel vector equilibrium problems, Comput. Appl. Math., 37, 1537-1549 (2018).

[3] Ding, X.P.: Existence and iterative algorithm of solutions for a class of bilevel generalized mixed equilibrium problems in Banach spaces, J. Glob. Optim. 53, 525-537 (2012)

[4] Fan, K.: A generalization of Tychonoff's fixed point theorem, Math Ann. 142, 305-310 (1961)

[5] Hai, N.X., Khanh, P.Q.: Existence of solution to general quasiequilibrium problem and applications, J. Optim. Theory Appl. 133, 317-327 (2007)

[6] Hung, N.V., O'Regan D.: Bilevel equilibrium problems with lower and upper bounds in locally convex Hausdorff topological vector spaces, Topology Appl. 269, 106939

[7] Hung, N.V., Hai, N.M.: Stability of approximating solutions to parametric bilevel vector equilibrium problems and applications, Comput. Appl. Math., 38, 17pp (2019) 
[8] Hung, N.V., Kobis, E., Tam, V.M.: Existence of solutions and iterative algorithms for weak vector quasi-equilibrium problems, J. Nonlinear Convex Anal. 21, 463-478 (2020)

[9] Hung, N.V., Tam, V.M., Kobis, E., Yao, J.C: Existence of solutions and algorithm for generalized vector quasi-complementarity problems with application to traffic network problems, J. Nonlinear Convex Anal. 20, 1751-1775 (2019)

[10] Hung, N.V., Tri, V.V., O'Regan D.: Existence conditions for solutions of bilevel vector equilibrium problems with application to traffic network problems with equilibrium constraints, Positivity, (2020), online first.

[11] Holmes, R.B.: Geometric Functional Analysis and its Application. SpringerVerlag, New York, (1975)

[12] Mordukhovich, B.S.: Equilibrium problems with equilibrium constraints via multiobjective optimization. Optim. Methods Softw. 19, 479-492 (2004) 